DE93010878

Presented at the 7th Meeting of the American Physical Society

Division of Particles and Fields

10-14 November, 1992

FERMILAB, llinois

\title{
Baryon Spectra in Au+Au Collisions: Preliminary Results From E-866
}

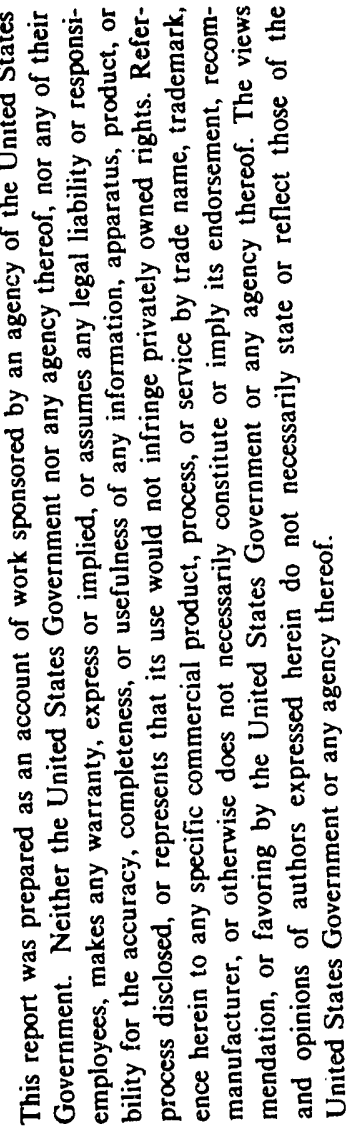

\author{
Michel GONIN \\ Physics Department, Brookhaven National Laboratory \\ Upton, N.Y. 11973, U.S.A. \\ for the E-802/E-866 Collaboration \\ $A N L, B N L, U C$-Berkeley, Columbia, LLNL, MIT, NYU, UC-Riverside \\ INS, Kyushu, Tokyo, Tsukuba
}

\begin{abstract}
Measurements are presented of single particle spectra from Au targets using the recently commissioned $A u$-beam at $11.6 \mathrm{GeV} / \mathrm{c}$ per nucleon from the BNL TandemBooster-AGS accelerator facilities. Protons and deuterons were detected with the 25msr E-802 spectrometer. Collision centrality was determined by measuring the forward energy in a calorimeter subtending a $1.2^{\circ}$ cone about $0^{\circ}$. Some comparisons with the cascade code ARC have been made.
\end{abstract}

\section{Introduction}

The Au-beam delivered recently to experiments at Brookhaven has provided the opportunity to achieve in a nuclear reaction the highest baryon densities ever obtained in any experiment. The data reported in this paper are from experiment E-866. The program of this experiment is to study semi-inclusive hadron spectra using magnetic spectrometers. This collaboration is a continuation of the E-802 experiment devoted to the study of relativistic heavy ion collisions with the proton, oxygen and silicon beams. Preliminary data concerning pion and kaon productions have been reported elsewhere ${ }^{1}$. One should note that beside the statistical errors shown in the following figures, a systematic error of $15 \%$ has to be applied for each preliminary $\mathrm{dN} / \mathrm{dy}$ point and inverse slope parameter.

\section{Detectors and triggers}

The existing E-802 magnetic spectrometer ${ }^{2}$ was used to make the measurements with the Au-beam. Due to the large particle multiplicities in Au+Au central collisions, the data reported here have been taken with the spectrometer positioned at backward angles $24^{\circ}, 34^{\circ}$ and $44^{\circ}$ where the multiplicities are compatible with 
the capabilities of the spectrometer.

An upgraded forward calorimeter (ZCAL) provided a centrality trigger. Figure 1 shows the corresponding kinetic energy spectra of the projectile spectators measured at zero degree in $\mathrm{Au}+\mathrm{Au}$. Note that the beam kinetic energy is 2108 $\mathrm{GeV}$. Software cuts on the spectra were used to define roughly the upper $4 \%$ of the total interaction cross section as shown in figure 1.

\section{Proton spectra}

The proton invariant cross section versus transverse kinetic energy are shown for central $A u+A u$ collisions in figure 2 from $y=0.55$ (top) to $y=1.35$ (bottom) with $\Delta y=0.1$. The $n^{\prime}$ th spectrum has been multiplied by $10^{-n}$. The spectra seem well described by an exponential function $\exp \left(-\mathrm{m}_{t} / \mathrm{T}\right)$ where $\mathrm{T}$ is called the inverse slope parameter. This function has been fit to the invariant spectra (solid lines). The multiplicity density $\mathrm{dN} / \mathrm{dy}$ has been obtained by integration of this function from 0 to $\infty$. In figure 3 are plotted $\mathrm{dN} / \mathrm{dy}$ and $\mathrm{T}$ distributions for central collision in $\mathrm{Au}+\mathrm{Au}(\simeq 4 \%)$ and $\mathrm{Si}+\mathrm{Al}(7 \%)$ reactions ${ }^{3}$ where reflected points about the center of mass rapidity are filled symbols. The horizontal axis represents the proton rapidity normalized to the beam rapidity $\mathrm{y} / \mathrm{y}_{\text {beam }}$ where $\mathrm{y}_{\text {beam }} \simeq 3.44$ for $\mathrm{Si}+\mathrm{Al}$ and $\mathrm{y}_{\text {beam }} \simeq 3.20$ for $\mathrm{Au}+\mathrm{Au}$. Quite different from the $\mathrm{Si}+\mathrm{Al}$ distribution, the proton $\mathrm{dN} / \mathrm{dy}$ distribution for $\mathrm{Au}+\mathrm{Au}$ shows no "target-like" peak at $\mathrm{y} / \mathrm{y}_{\text {beam }} \simeq$ 0.7. This suggest that a fairly large amount of "stopping" has been achieved in central $\mathrm{Au}+\mathrm{Au}$ collisions. The inverse slope parameter is broader for the heavier system probably due to the larger number of collisions. Thus the incident energy seems more thermalized in the $\mathrm{Au}+\mathrm{Au}$ reaction than in the $\mathrm{Si}+\mathrm{Al}$ reaction.

\section{Deuteron production}

Emission of deuterons in heavy ion collisions at lower energies has been described successfully by the coalescence model ${ }^{4}$. According to this simple model, the probability of forming a deuteron is the highest when a proton and a neutron are located within an interaction region in the phase-space. In figure 4 the $\mathrm{d} / \mathrm{p}^{2}$ multiplicity ratios are shown versus rapidity for $\mathrm{Si}+\mathrm{Al}^{3}$ (peripheral and central collisions) and $A u+A u$ (central collisions). This ratio is almost independent of the rapidity which is consistent with the coalescence picture but seems sensitive to the number of nucleons involved in the collision. From these ratios and by using a thermal model ${ }^{5}$, one can estimate the size of the interaction region. The radii $R$ of the equivalent spheres are plotted in figure 4 versus rapidity. The values increase for $\mathrm{R} \simeq 4.2 \mathrm{fm}$ for $\mathrm{Si}+\mathrm{Al}$ peripheral to $\mathrm{R} \simeq 6.6 \mathrm{fm}$ for $\mathrm{Si}+\mathrm{Al}$ central and $\mathrm{R} \simeq 8.4$ $\mathrm{fm}$ for $\mathrm{Au}+\mathrm{Au}$ central. The fact that these radii are larger than the radij of the projectiles suggest that deuteron are probably produced during the latest stages of the collision to avoiding any breakup into individual nucleons or that the thermal 
model is inadequate for ultra-relativistic heavy-ion collisions.

\section{Comparison with cascade calculations}

The preliminary proton data have been compared to the prediction of the relativistic cascade code $A R C{ }^{5}$ for $A u+A u$ collisions at impact parameter $b \leq 3 \mathrm{fm}$. Note that this condition ( $b \leq 3 \mathrm{fm}$ ) represents an approximation of the experimental centrality cut. Figure 5 shows the proton $\mathrm{dN} / \mathrm{dy}$ distribution and the inverse slope parameter versus rapidity. The lines represent the ARC calculations. As it can be seen, the agreement between the data and the model is excellent. A small difference is observed between the model and the experimental inverse slope parameter. Invariant spectra for proton in the rapidity interval $y=[1.0-1.2]$ are shown in figure 6 . It is shown that the small discrepancy observed between the code and the data occurs at low and high $\mathrm{m}_{t}$. Since the code ARC relies heavily on production of resonances ( $\Delta$ 's, $\rho$ 's), the excellent agreement between the code and the data suggests that resonances might play a leading role in the dynamics of the $\mathrm{Au}+\mathrm{Au}$ collisions at $11.6 \mathrm{~A} . \mathrm{GeV} / \mathrm{c}$.

\section{Acknowledgements}

We are pleased to acknowledge the expertise of the AGS-Tandem staff for having delivured the gold beam on time. The authors also thanks T.J. Shlagel, S.H. $\mathrm{K}$ s.hana and $\mathrm{Y}$. Pang for allowing them to show the ARC predictions. Experiments 802,866 are supported in part by the U.S. Department of Energy contracts and grants with ANL, BNL, UC-Berkeley, UC-Riverside, Columbia, LLNL, and MIT, in part by NASA under contract with UC-Berkeley, and by the US-Japan High Energy Physics Collaboration Treaty.

\section{References}

1. M. Gonin, E-802/E-866 collaborations, INPC, Wiesbaden, July 1992

2. T. Abbott et al. E-802 collaboration, NIM A290 (1990) 41

3. T. Abbott et al. E-802 collaboration, to be published

4. S.F. Butler and C.A. Pearson, Phys. Rev. 128 (1963) 836

5. L.P. Csernai and J.I. Kapusta, Phys. Rep. 131 (1986) 223

6. Y. Pang et al., Phys. Rev. Lett. 68 (1992) 2743

RECEIVED

APR 071993

OSTI 


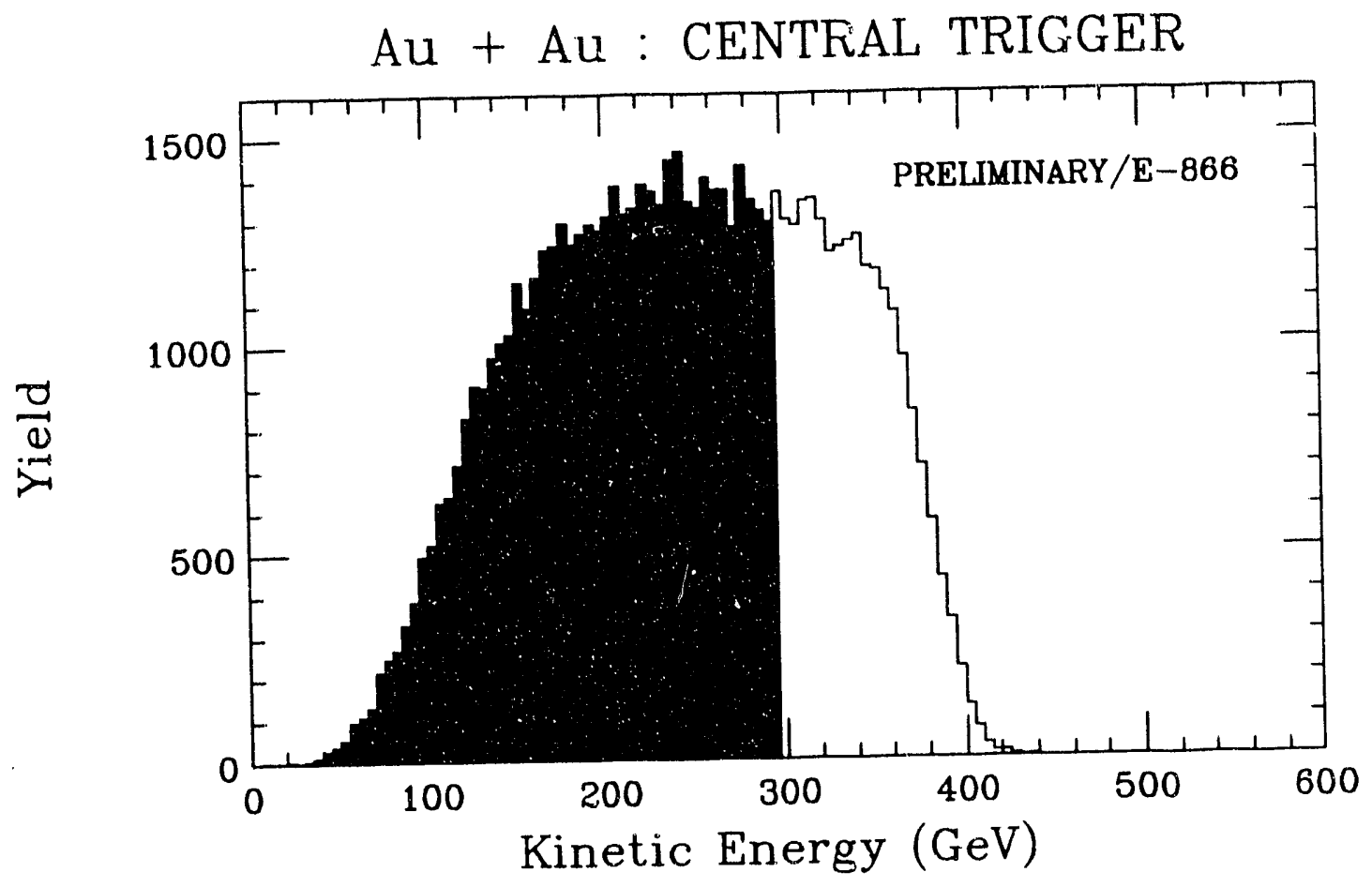

Fig 1: kinetic energy spectra of projectile spectators

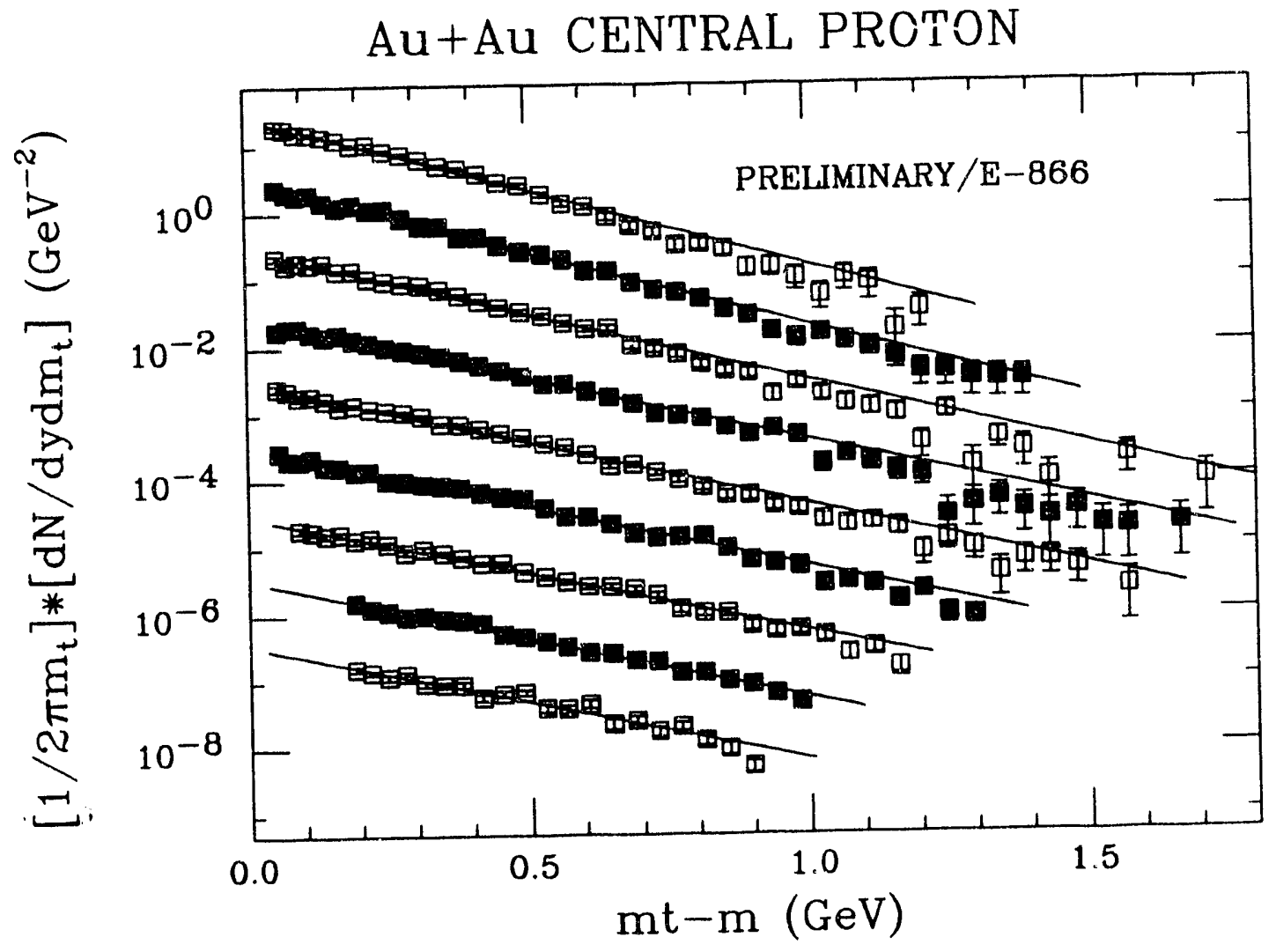

Fig 2: invariant cross section for proton. See texte 


\section{Proton CENTRAL}

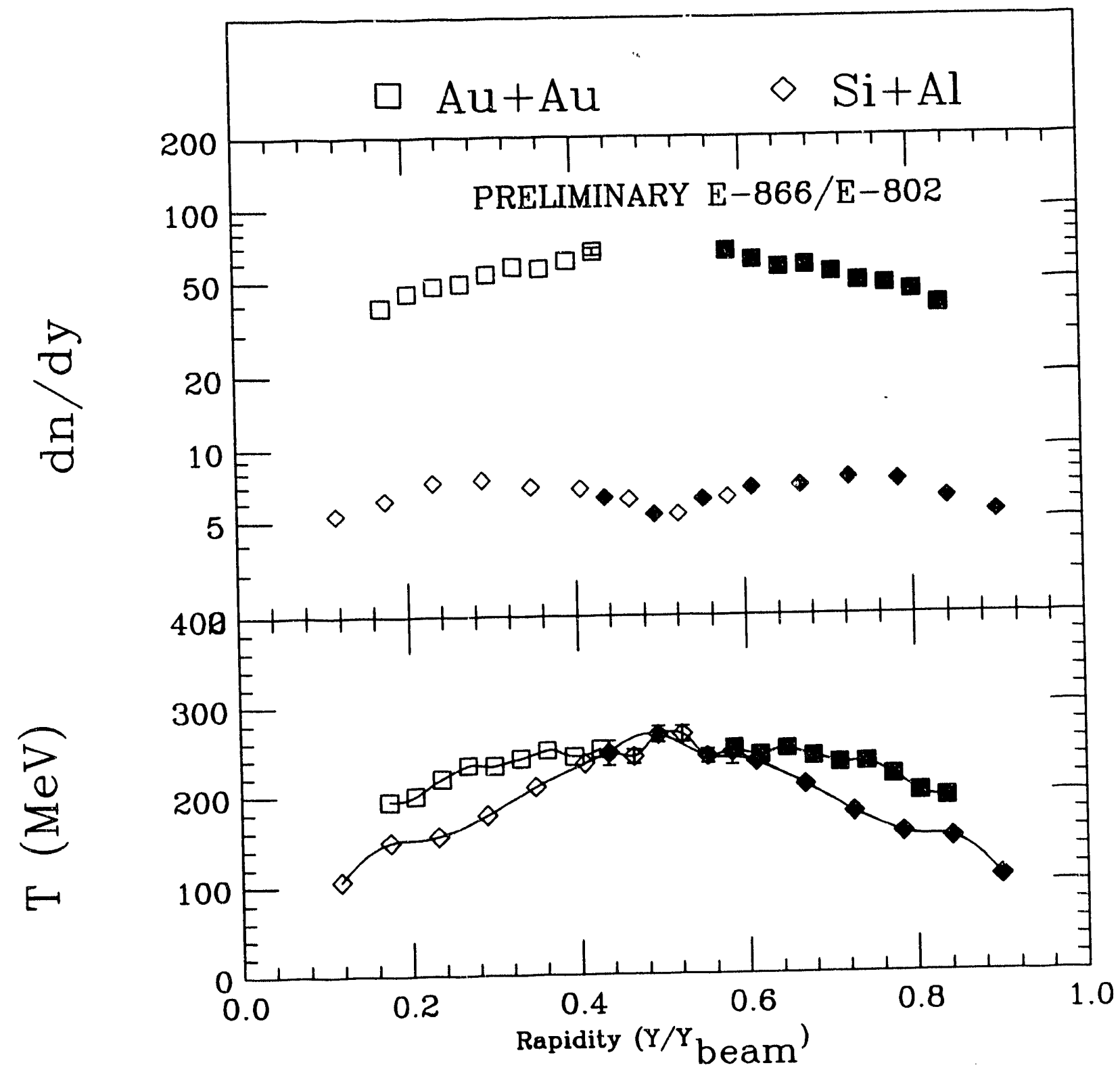

Fig.3: Proton data for central $\mathrm{Au}+\mathrm{Au}$ (11.6 A.GeV/c) and $\mathrm{Si}+\mathrm{Al}(14.6 \mathrm{~A} . \mathrm{GeV} / \mathrm{c})$ 


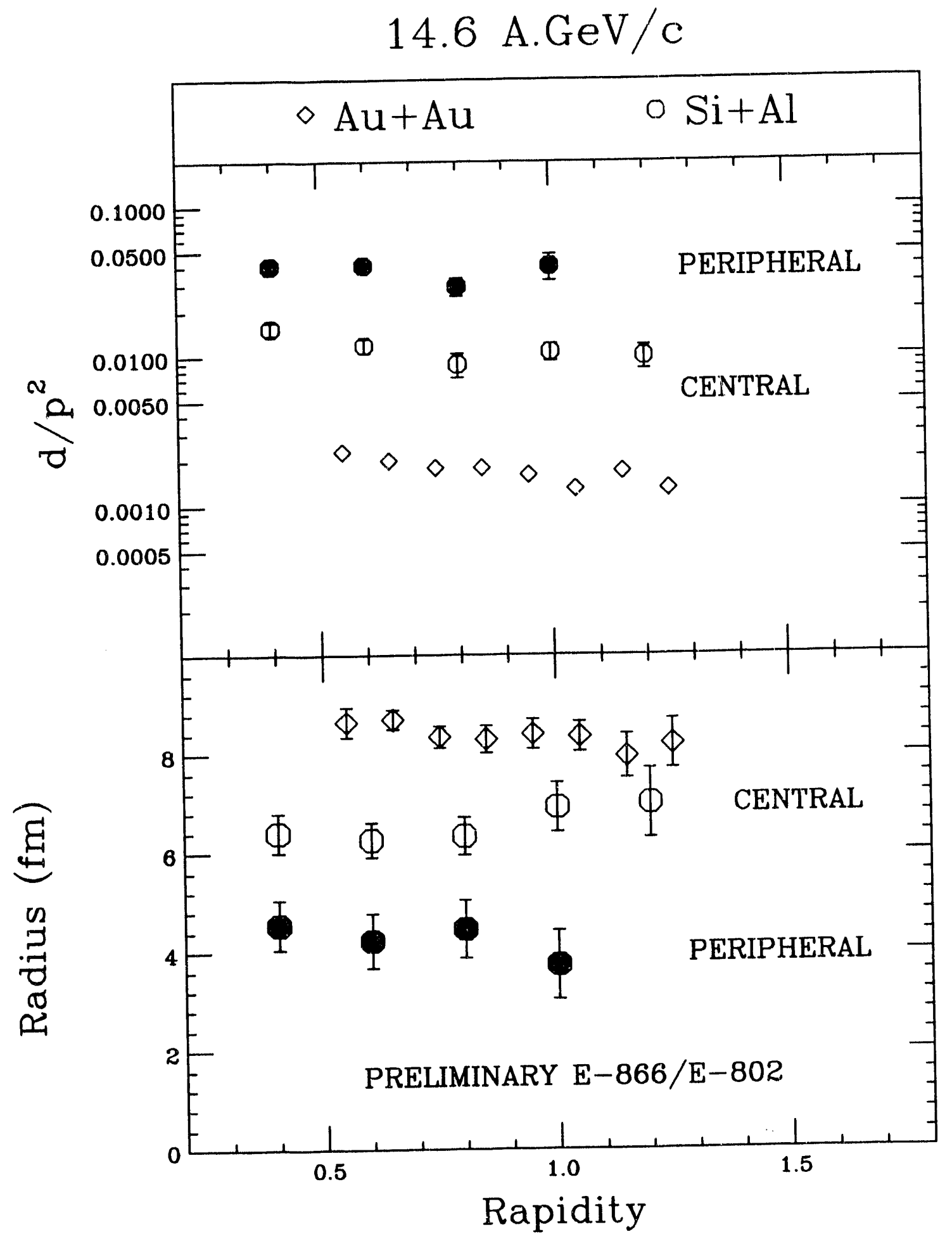

Fig.4: Ratio $\mathrm{d} / \mathrm{p}^{2}$ plotted as a function of rapidity. Radii of the interaction region deduced from $d / p^{2}$ 


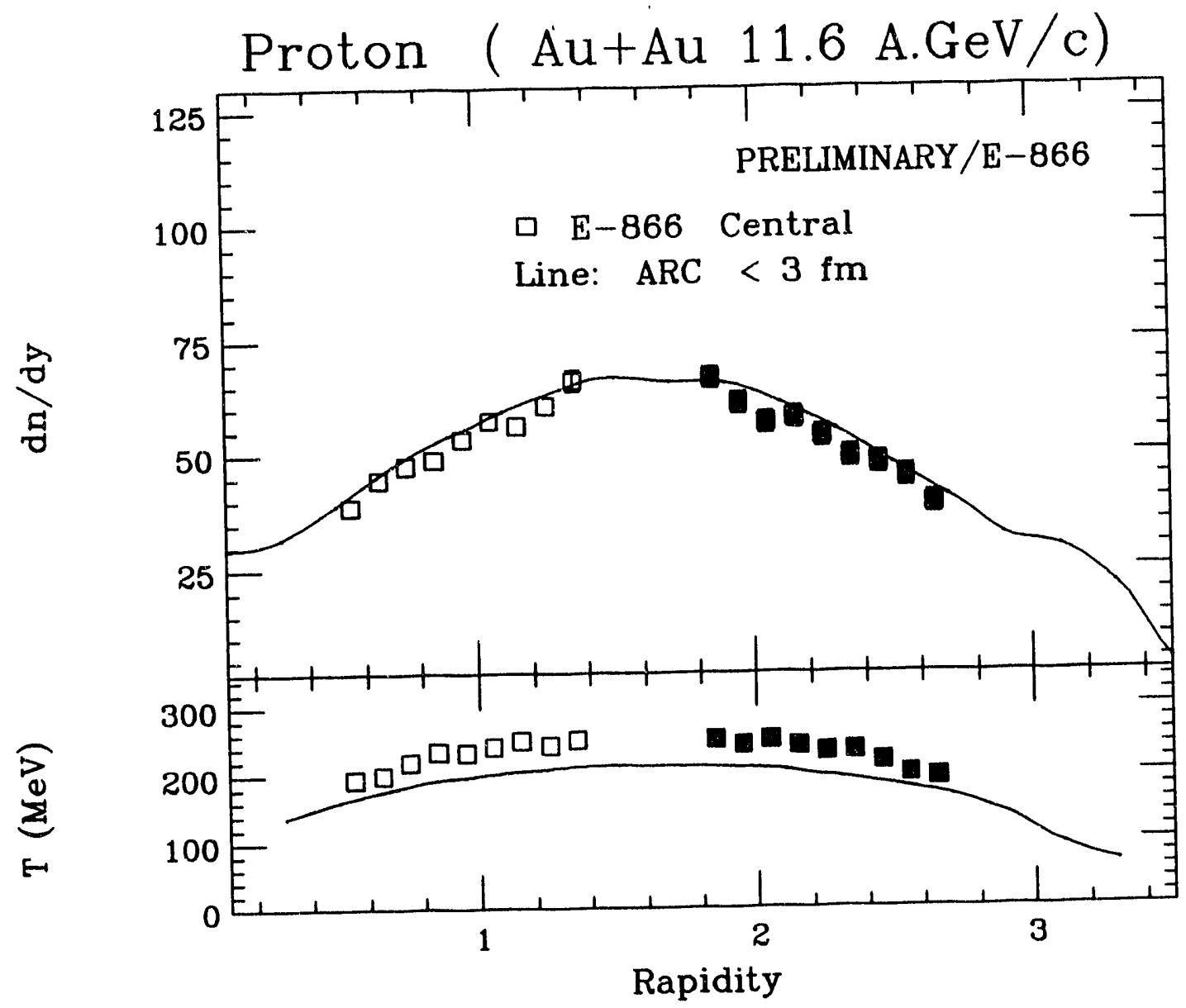

Fig.5: ARC predictions are compared to E-866 proton data

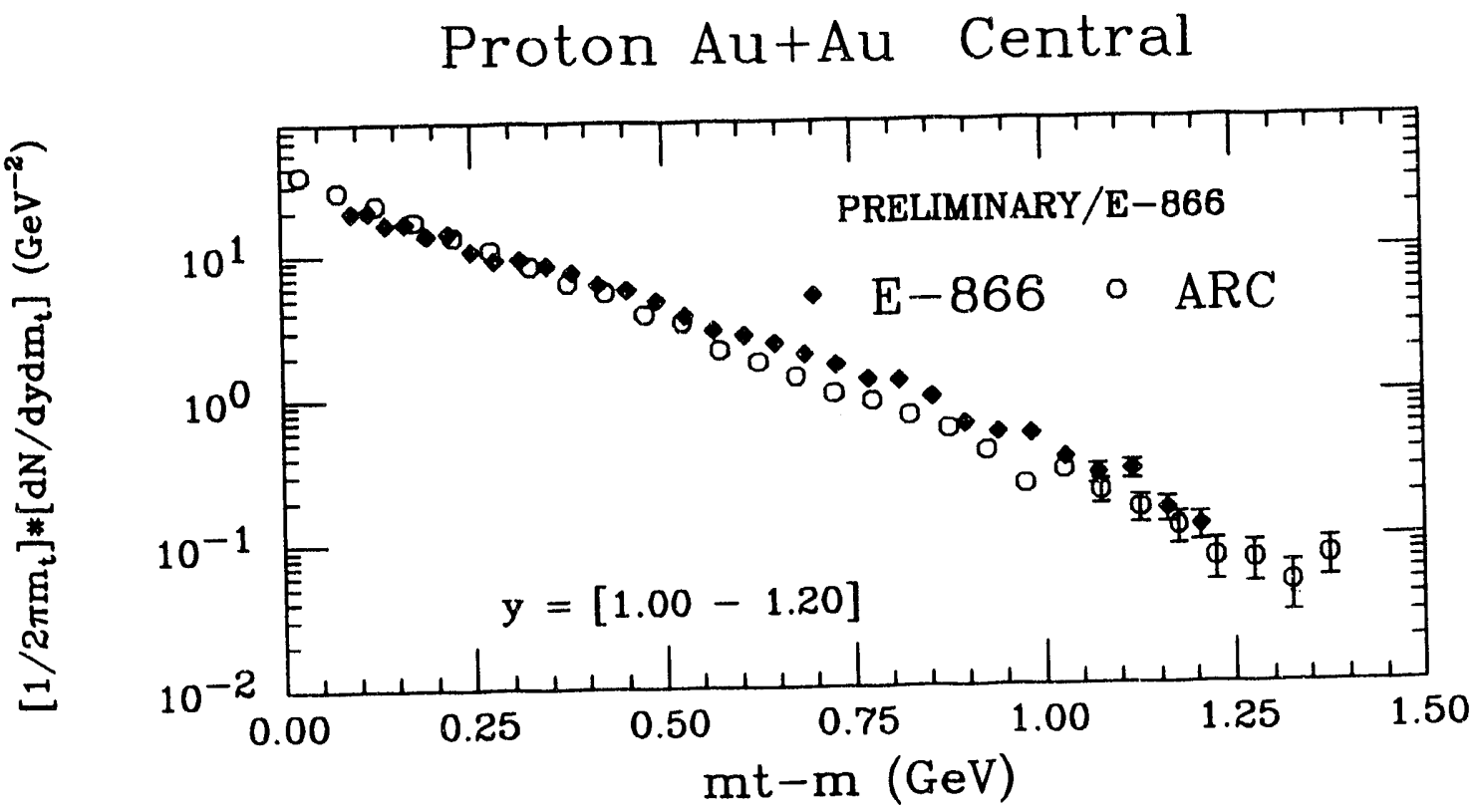

Fig.6: invariant spectra for proton for $y=[1.0-1.2]$ 

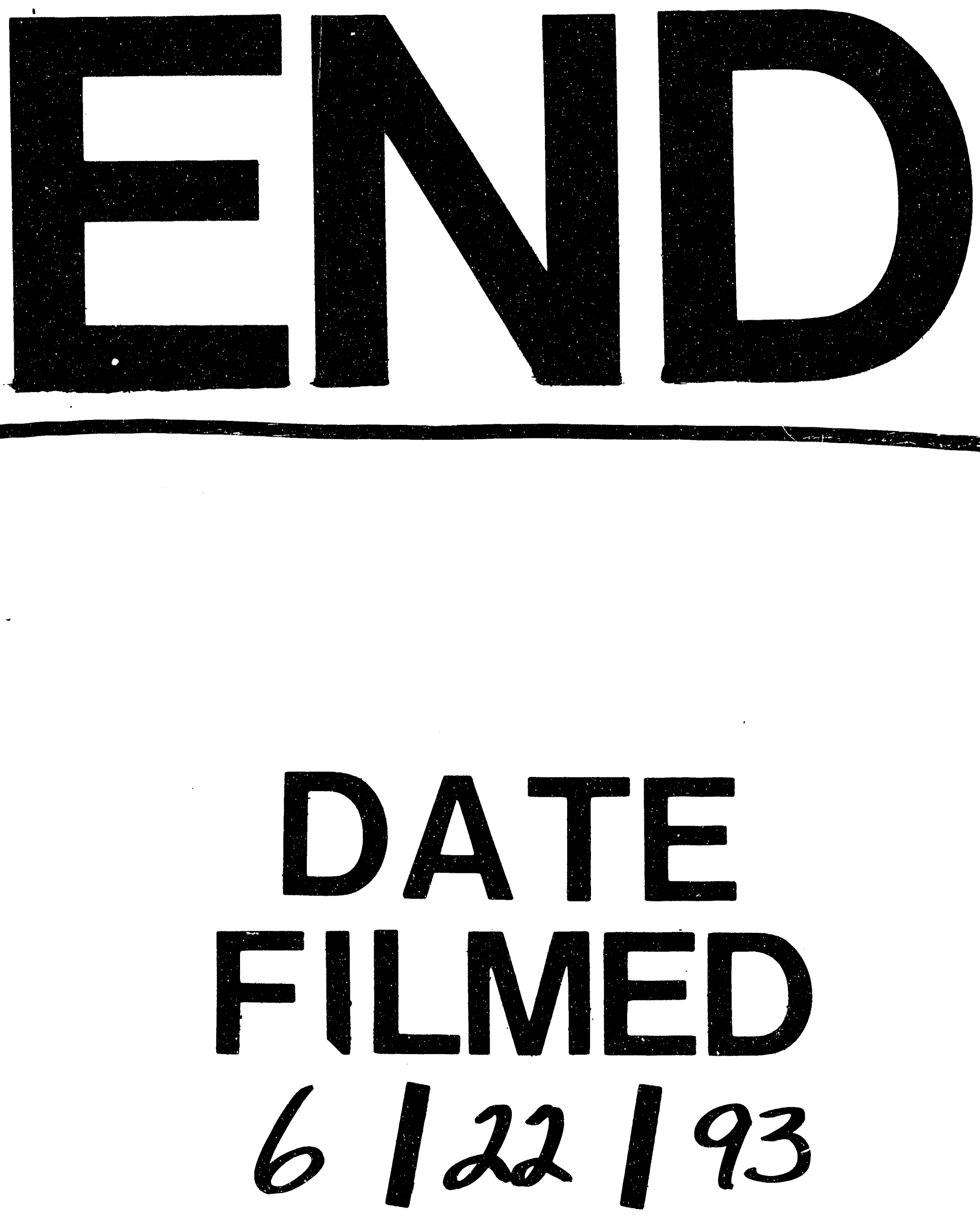
